\title{
Análise facial frontal em repouso e durante o sorriso em fotografias padronizadas. Parte I - Avaliação em repouso*
}

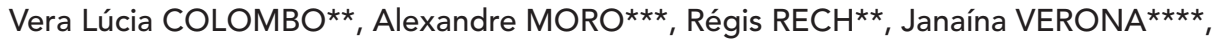 \\ Gilce C. Alves da COSTA ${ }^{\star \star \star \star \star}$
}

\begin{abstract}
Resumo
Considerando a importância da análise facial frontal no diagnóstico, esse trabalho propõem-se a apresentar uma análise facial frontal em fotografias padronizadas, em repouso e durante o sorriso, que possa auxiliar no diagnóstico e planejamento do tratamento ortodôntico e cirúrgico como também na avaliação dos resultados obtidos. Fizeram parte da amostra estudada 40 mulheres, com idade média de 22 anos, leucodermas, com faces agradáveis, Classe I de Angle, sem história prévia de tratamento ortodôntico e/ou cirurgia plástica na face. Foram obtidas fotografias faciais frontais $(10 \mathrm{x} 15 \mathrm{~cm}$ coloridas), padronizadas. Em repouso e durante o sorriso máximo. Sobre as fotografias, foram realizadas medidas lineares, angulares e proporcionais. As medidas foram avaliadas por meio de teste de normalidade, estatística descritiva e desvio padrão do erro. Os resultados mostraram que algumas das medidas utilizadas em nosso trabalho se assemelham às encontradas na literatura e outras diferem muito. E a partir desse estudo propõe-se uma análise facial frontal para utilização no diagnóstico e planejamento do tratamento ortodôntico e cirúrgico.
\end{abstract}

Palavras-chave: Análise facial frontal. Ortodontia. Estética facial.

\section{INTRODUÇÃO}

A literatura ortodôntica e odontológica de modo geral está repleta de trabalhos sobre estética facial. Existem muitas análises faciais, entretanto, a grande maioria está voltada para o perfil do paciente e apenas algumas preocupam-se com a vista frontal, faltando assim, a visão do conjunto. O desenvolvimento de técnicas de telerradiografias de perfil e a importância da dimensão ântero-posterior na má oclusão têm a atenção focalizada sobre o aspecto lateral em detrimento do aspecto frontal. Entretanto, são necessárias informações referentes ao relacionamento dos tecidos moles e dentes em uma visão frontal para determinar uma base ampla de avaliação da estética facial.

\footnotetext{
* Resumo da Monografia apresentada ao Curso de Especialização em Ortodontia e Ortopedia Facial da EAP-ABO/PR, como requisito para obtenção do título de Especialista.

** Especialista em Ortodontia e Ortopedia Facial pela EAP-ABO/PR

*** Mestre e Doutor em Ortodontia, professor Adjunto do Departamento de Anatomia da UFPR, professor dos Cursos de Especialização em Ortodontia e Ortopedia Facial da EAP-ABO/PR e UFPR.

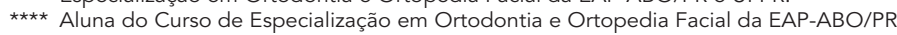

***** Especialista em Ortodontia e Ortopedia Facial, Professora do Curso de Especialização em Ortodontia e Ortopedia Facial da EAP-ABO/PR.
} 
Partindo do princípio de que o esqueleto da face de uma pessoa não é visto durante o exame clínico, parece razoável e lógico que um tempo maior seja despendido na avaliação e planejamento do tratamento a partir daquilo que podemos ver. De acordo com Mackley ${ }^{32}$, é o tecido mole da face que nós vemos, no dia-a-dia, durante o diálogo com as pessoas. É de uma visão frontal que as pessoas fazem uma auto-avaliação da sua estética facial.

Freqüentemente, a necessidade estética motiva o paciente a procurar o tratamento ortodôntico. O paciente deve ser tratado sob o ponto de vista estético e não somente sob o ponto de vista cefalométrico e funcional. $\mathrm{O}$ tratamento deve ter um objetivo oclusal que combine com a estética facial.

$\mathrm{Na}$ documentação ortodôntica, as fotografias da face são analisadas somente do ponto de vista qualitativo, raramente é feita uma avaliação quantitativa. Com uma técnica padronizada, a análise fotográfica da face poderá ser usada, juntamente com a análise radiográfica convencional, no diagnóstico e planejamento de tratamento ortodôntico e cirúrgico.

Assim, este trabalho tem por objetivo elucidar as seguintes questões:

a) quais são os padrões médios de normalidade para as variáveis da análise da fotografia frontal em repouso da amostra estudada?

b) as medidas obtidas nesta amostra assemelhamse às medidas encontradas na literatura?

c) do ponto de vista da repetibilidade, as variáveis são confiáveis?

d) propor uma análise facial frontal em fotografias padronizadas.

\section{REVISÃO DA LITERATURA}

A estética facial tem sido discutida desde a antigüidade. Naquela época, os conceitos de estética dependiam mais da preferência tradicional ou pessoal. Ricketts ${ }^{42}$ descreveu a estética como o estudo da beleza e, juntamente com a ética, a política, a lógica, e a metafísica, seria uma ramificação da filosofia básica. Dierkes ${ }^{16}$ afirma que a beleza da face pode ser definida como um estado de har- monia, um equilíbrio entre as proporções faciais, um relacionamento de equilíbrio entre as estruturas esqueléticas, os dentes e os tecidos moles.

Muitos autores avaliaram a estética facial através de medidas feitas diretamente na face ${ }^{1,2,4,19,20,21,22,23,47}$, através de fotografias ${ }^{5,7,8,9,10,24,30,33,38,41,44,45}$ técnica estereométrica ${ }^{3,25,26,27}$ ou por meio de técnicas de varredura a laser ${ }^{35}$ e técnicas computadorizadas ${ }^{18,36,37}$.

Para Gonzáles-Ulloa ${ }^{29}$ é impossível estabelecer um padrão de beleza universal devido aos diferentes tipos étnicos ou idade, mas em cada face bela, apesar da origem étnica, existe proporção e harmonia entre os segmentos. A beleza reflete as particularidades culturais de um povo, da região onde vive, num período determinado de tempo ${ }^{41}$.

A fotografia padronizada, enfatizada por diversos autores $6,7,8,12,24,28,46,50$, permite avaliar detalhadamente medidas e proporções, o que seria difícil realizar diretamente na face.

A fotografia da face é uma ferramenta importante no diagnóstico e planejamento ortodôntico. É de baixo custo, não expõe o paciente à radiação nociva, fornece melhor avaliação do relacionamento harmônico entre as estruturas craniofaciais internas e externas, incluindo a contribuição dos tecidos adiposos ${ }^{24}$ e diminui a necessidade da presença do paciente durante a avaliação. Nas fotografias pré-tratamento podemos avaliar os pontos positivos que deverão ser mantidos e os que deverão ser modificados para obtermos uma melhora na estética facial.

As fotografias neste estudo foram realizadas com o indivíduo na posição natural da cabeça $^{1,2,11,14,34,48,49}$. Esta é a posição em que o indivíduo se encontra no dia-a-dia e está relacionada com a posição correta natural do corpo e o alinhamento da coluna cervical, baseia-se na linha da visão e é determinada pelo equilíbrio geral da cabeça e do corpo quando o indivíduo olha reto para a frente ${ }^{48}$. Nesta posição, as pupilas ficam centradas no meio dos olhos, definindo a linha de visão ou a horizontal verdadeira, devendo esta ficar paralela ao solo.

O plano horizontal de Frankfurt é utilizado por alguns autores para posicionar a cabeça do indiví- 
duo na tomada fotográfica ${ }^{7,8,9}$. Segundo Arnett e Bergman $^{1,2}$ o plano horizontal de Frankfurt no indivíduo não é paralelo ao solo, conseqüentemente não deve determinar a posição da cabeça na análise facial. Para Moorrees ${ }^{34}$, a inclinação de todas as linhas de referências intracranianas estão sujeitas a variações biológicas; portanto, elas não são adequadas para análises cefalométricas precisas.

Vários autores avaliaram a reprodutibilidade da posição natural da cabeça $a^{11,13,14,48}$. Segundo Cooke e Wei ${ }^{14}$, a reprodutibilidade da posição natural da cabeça é melhor com o uso do espelho, enquanto Chiu e Clark ${ }^{11}$, utilizando uma linha vertical verdadeira, não encontraram diferença estatisticamente significante entre o uso ou não do espelho.

Segundo Viazis ${ }^{48}$, o tempo para que o indivíduo posicione a cabeça em sua posição natural não deverá ser maior do que 3 minutos, e é o profissional quem determina a posição final da cabeça, corrigindo-a de forma a evitar que o indivíduo incline ou estenda sua cabeça em posição "não natural".

\section{MATERIAL E MÉTODO}

\section{Material}

Fizeram parte da amostra estudada 40 indivíduos do gênero feminino, leucodermas, com idade entre 18 e 28 anos (média de 22 anos). Na obtenção da fotografia, foi utilizada uma máquina fotográfica Canon EOS Rebel X, lente macro 100/35 mm Canon, flash circular Canon, tripé Zenit TZ-40, filme Kodak, colorido, ASA 100/35 mm, um espelho, um fio com um peso na extremidade (utilizado como referência vertical) e 80 fotografias coloridas, tamanho $10 \times 15 \mathrm{~cm}$.

\section{Método \\ Fotografia}

De todos os indivíduos foram realizadas fotografias frontais da face. Cada indivíduo foi fotografado sentado, olhando diretamente para seus olhos refletidos em um espelho colocado à sua frente ${ }^{24}$. $\mathrm{O}$ indivíduo foi instruído a manter uma postura ereta, "natural e normal", com ambos os braços livres ao lado do tronco. Essa posição corresponde à "posição natural de cabeça" de Broca ${ }^{24}$. Na fotografia em repouso, os lábios estavam em posição relaxada e levemente selados. Para termos uma referência vertical verdadeira, foi usado um fio preso ao teto, caindo ao lado da cabeça e com um peso em sua extremidade ${ }^{11}$ visível na fotografia.

A distância focal utilizada foi de um metro (dada pela regulagem da lente macro 100/35 mm), a velocidade de 60 e a abertura da lente de 11 . Para permitir uma correlação entre as medidas reais e as medidas obtidas nas fotografias, em 10 indivíduos foram marcados dois pontos na testa com uma distância de $1 \mathrm{~cm}$ entre eles. Também foi fotografada uma régua, nos mesmos padrões da fotografia da face. A medida de $0,55 \mathrm{~mm}$ da fotografia corresponde aproximadamente a $1 \mathrm{~cm}$ do tamanho real da face, isto é, a fotografia corresponde a 55\% do tamanho real. A distorção é dada em números aproximados, já que, na fotografia, temos uma visão bidimensional da estrutura tridimensional da face.

Todos os filmes fotográficos foram revelados num mesmo laboratório Kodak, padrão QLab (padrão de qualidade internacional Kodak).

\section{SELEÇÃO DA AMOSTRA}

A amostra foi obtida nos estados do sul do Brasil e uma banca composta por professores e alunos do Curso de Especialização em Ortodontia e Ortopedia Facial da ABO-PR selecionou 40 faces de indivíduos do gênero feminino, leucodermas, filhas de pais brasileiros de origem européia (italianos, alemães, portugueses, holandeses e espanhóis), com idade entre 18 e 28 anos, a partir de uma amostra prévia de 83 indivíduos. Esses indivíduos apresentavam os seguintes requisitos:

a) possuíam uma oclusão de Classe I (aceitou-se um apinhamento mínimo ou pequenos diastemas que não justificassem um tratamento ortodôntico);

b) apresentavam uma face agradável;

c) não tinham sido tratados indivíduos ortodonticamente;

c) não tinham se submetido a nenhum tipo de cirurgia plástica facial. 


\section{TRAÇADO FOTOMÉTRICO Desenho anatômico}

O desenho anatômico da face em repouso foi realizado de forma que se permitisse o traçado dos pontos e linhas que seriam utilizados.

\section{Pontos fotométricos utilizados (Fig. 1)}

Gl' - Glabela tegumentar - ponto mais proeminente da linha média sobre a testa ${ }^{18}$.

N' - Násio tegumentar - ponto mais côncavo da linha média sobre a porção frontal da ponte do nariz ${ }^{18}$.

Para demarcação dos pontos GL' e N' foi utilizada uma fotografia de perfil obtida com a mesma padronização das fotografias frontais. $\mathrm{Na}$ fotografia de perfil, foram marcados os pontos Gl, $\mathrm{N}$ e Ex. Esses pontos foram transferidos para a linha vertical verdadeira e a medida de distância entre eles foi obtida. Essa distância foi transferida para a fotografia frontal, tomando como base a linha interexocanto (Exd-Exe).

Ponto V - Ponto sobre a linha que passa pelo exocanto direito e esquerdo (Exd-Exe), eqüidistante dos pontos endocanto direito e esquerdo (End e Ene).

Ex - Exocanto direito (Exd) e esquerdo (Exe) ponto no canto lateral do olho direito e esquerdo ${ }^{40}$

Pn - Ponta do nariz - ponto central da ponta do nariz, determinado por inspeção visual.

Sn - Subnasal - ponto localizado na confluência da margem inferior da base do nariz com lábio superior. Ponto onde a borda inferior do septo nasal se une à raiz do lábio superior ${ }^{17}$.

F - Filtro inferior - ponto mais inferior do filtro onde este intersecta com o vermelhão do lábio superior ${ }^{40}$.

Ls - Lábio superior - ponto médio da junção cutânea com o vermelhão do lábio superior ${ }^{19}$.

$\mathrm{Ab}$ - Ângulo da boca do lado direito (Abd) e esquerdo (Abe) - ponto no canto mais externo da boca na junção do vermelhão do lábio com a pele do lado direito e esquerdo.

Es - Estômio - ponto sobre a linha média facial na união entre o vermelhão do lábio superior com o inferior.
Li - Lábio inferior - ponto médio da junção cutânea com o vermelhão do lábio inferior ${ }^{19}$.

Zi' - Zígio direito (Zid') e esquerdo (Zie') - ponto mais lateral do arco zigomático do lado direito e esquerdo.

Go' - Gônio direito (God') e esquerdo (Goe') - ponto mais externo e proeminente do ângulo da mandíbula do lado direito e esquerdo ${ }^{18}$.

Me' - Mentoniano - ponto mais inferior sobre o contorno do tecido mole que cobre a sínfise mandibular ${ }^{33}$.

\section{Linhas fotométricas utilizadas}

Linha interexocanto - Linha horizontal que de estende do exocanto direito (Exd) ao exocanto esquerdo (Exe).

Linha média facial - Linha traçada verticalmente passando pelo ponto násio (N') e o filtro inferior $(\mathrm{F})$.

Linha subnasal - mentoniano - Linha que se estende do ponto subnasal (Sn) até o ponto mentoniano (Me').

Linha V-God' - Linha oblíqua que une os pontos $\mathrm{V}$ e gônio direito (God').

Linha V-Goe' - Linha oblíqua que une os pontos $V$ e gônio esquerdo (Goe').

Linha Me'-Exd - Linha oblíqua que se estende do ponto mentoniano (Me') ao exocanto direito (Exd).

Linha Me'-Exe - Linha oblíqua que se estende do ponto mentoniano (Me') ao exocanto esquerdo (Exe).

Linha da comissura - Linha horizontal que se estende do ângulo da boca no lado direito (Abd) ao ângulo da boca no lado esquerdo (Abe).

Linha Zid'-God' - Linha oblíqua que une os pontos zigomático direito (Zid') e gônio direito (God').

Linha Zie'-Goe' - Linha oblíqua que une os pontos zigomático esquerdo (Zie') e gônio esquerdo (Goe').

Linha Exd-God' - Linha oblíqua que une os pontos exocanto direito (Exd) e gônio direito (God').

Linha Exd-Goe' - Linha oblíqua que une os pontos exocanto esquerdo (Exe) e gônio esquerdo (Goe') 


\section{Medidas fotométricas lineares utilizadas (Fig. 2)}

Comprimento do lábio superior (Sn-Es) -Distância do subnasal (Sn) ao estômio (Es).

Comprimento lábio inferior-mentoniano (Es-Me') - Distância do estômio (Es) ao mentoniano (Me').

Extensão do vermelhão do lábio superior (Ls-Es) - Distância do ponto médio da junção cutânea com o vermelhão do lábio superior (Ls) ao estômio (Es).

Extensão do vermelhão do lábio inferior (Es-Li) Distância do estômio (Es) ao ponto médio da junção cutânea com o vermelhão do lábio inferior (Li).

Altura da boca (Ls-Li) - Distância do ponto médio da junção cutânea do lábio superior (Ls) ao ponto médio da junção cutânea do lábio inferior (Li).

Inclinação da linha da comissura - É a diferença, em milímetros, entre a distância da linha da comissura à linha interexocanto na altura do ângulo da boca direito (Abd) e a distância da linha da comissura à linha interexocanto na altura do ângulo da boca esquerdo (Abe).

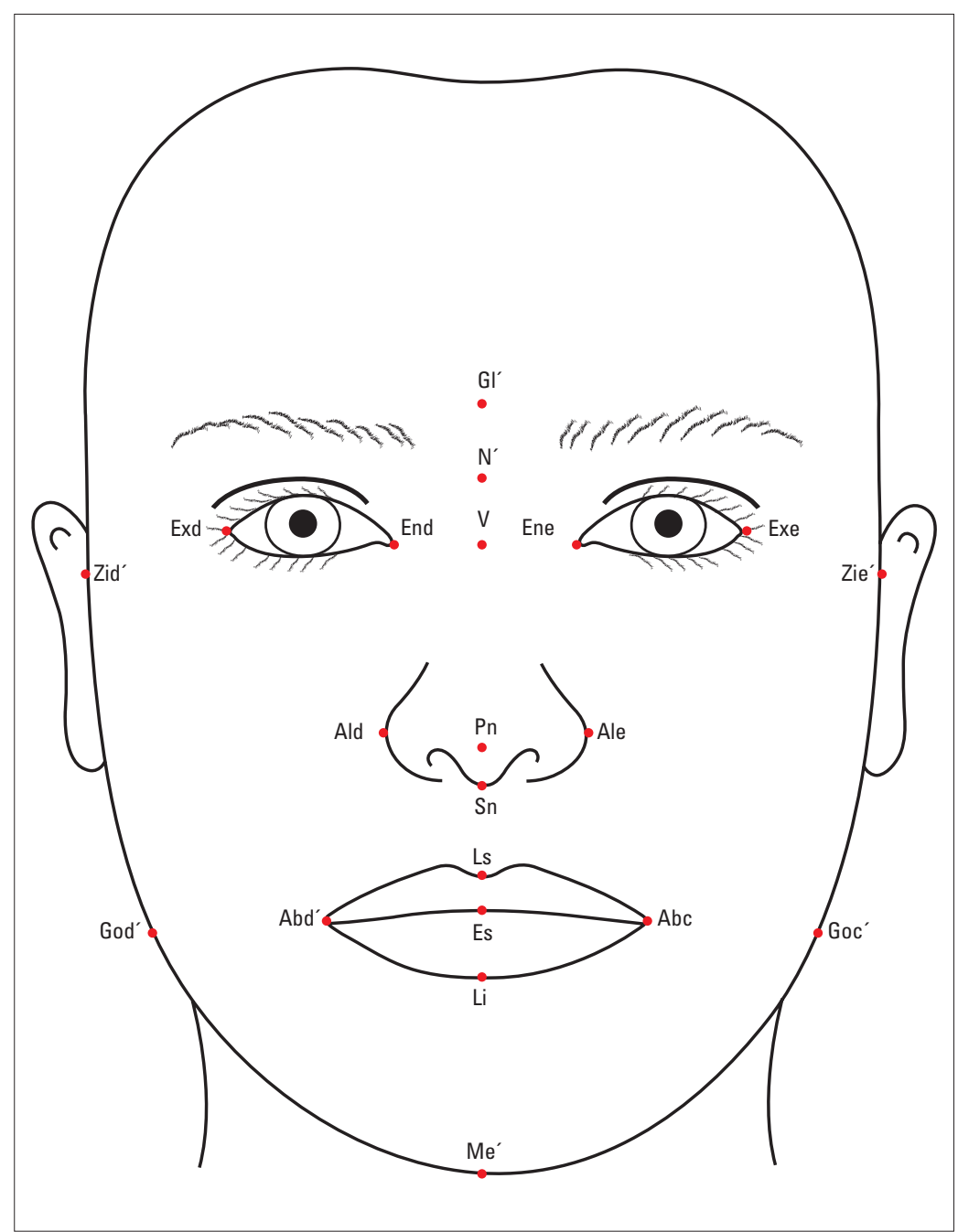

FIGURA 1 - Pontos fotométricos.

LEGENDA: GI' - glabela; N' - Násio; V - ponto V; Exd - exocanto direito; Exe - exocanto esquerdo; Pn - ponta do nariz; Sn - subnasal; $\mathbf{F}$ - filtro inferior*; Ls - lábio superior; Abd - âng. da boca dir; $\mathbf{A b e}$ - âng. da boca esq.; Es - estômio; End - endocanto direito; Li - lábio inferior; Zid' - zígio direito; Zie' - zígio esquerdo; God' - gônio direito; Ald - alar direito; Goe' - gônio esquerdo; Me' - mentoniano; Ene - endocanto esquerdo; $\mathbf{A l e}$ - alar esquerdo; $\mathbf{F}$ - (filtro) coincidente com o ponto Ls ( lábio superior).

*F - (filtro) coincidente com o ponto Ls (lábio superior). 
Largura da boca (Abd-Abe) - Distância linear do ângulo da boca direito (Abd) ao ângulo da boca esquerdo (Abe).

Alinhamento da linha média facial - São observadas as posições relativas dos pontos do tecido mole, ponta do nariz e ponto mentoniano, em relação à linha média facial.

\section{Medidas fotométricas proporcionais utiliza- das (Fig. 2)}

Índice facial - É a proporção entre a altura da face (N'-Me') e a largura da face superior (Zid'-Zie'). O índice facial é calculado pela seguinte fórmula ${ }^{17}$ :

$$
\frac{\text { altura facial x } 100}{\text { largura bizigomática }}
$$

Este índice mostra se a face é estreita e longa, dolicofacial (valores maiores que 90.0), ou curta e larga, braquifacial (valores menores que 85.0). O tipo médio é o mesofacial (valores entre 85.0 - 89.9).

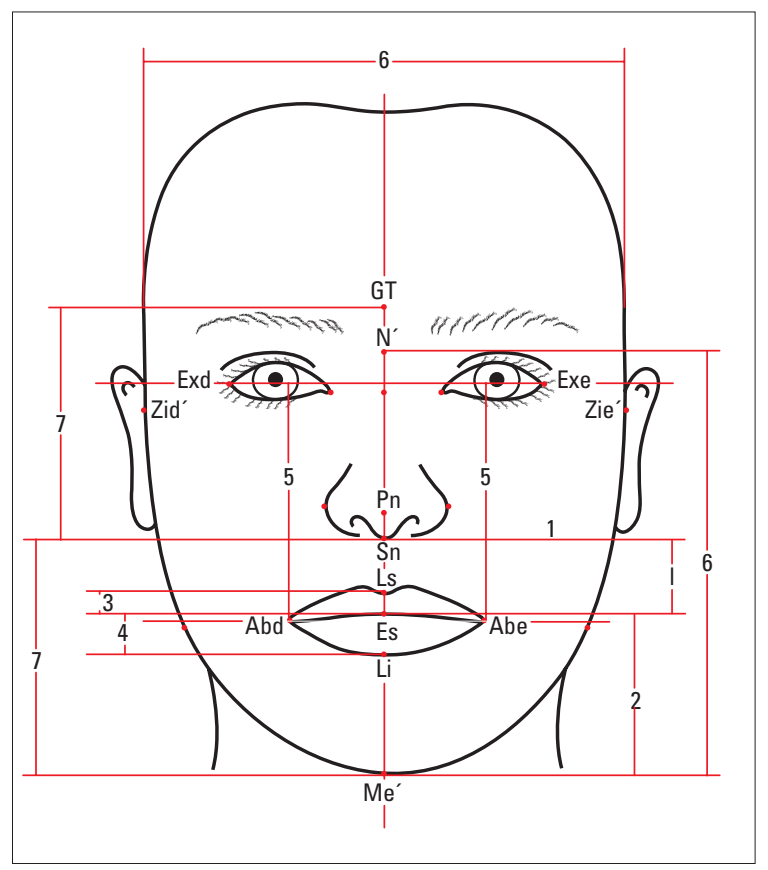

FIGURA 2 - Medidas fotométricas lineares e proporcionais.

01 - Comprimento do lábio superior (Sn-Es)

02 - Comprimento lábio inferior-mentoniano (Es-Me')

03 - Extensão do vermelhão do lábio superior (Ls-Es)

04 - Extensão do vermelhão do lábio inferior (Es-Li)

05 - Inclinação da linha da comissura

06 - Índice facial

07 - Altura da face média (Gl'-Sn) : Altura da face inferior (Sn-Me')
Proporção da altura facial - Proporção entre a altura da face média (Gl'-Sn) e a altura da face inferior (Sn-Me').

\section{Medidas fotométricas angulares utilizadas} (Fig. 3)

Ângulo da simetria facial - Ângulo formado entre a linha média da face e a linha subnasalmentoniano.

Ângulo da abertura facial modificado - Ângulo formado pelas linhas da direita e da esquerda, que se estendem desde os pontos mais laterais das órbitas direita e esquerda (Exd e Exe) até o ponto mentoniano (Me'). A intersecção destas duas linhas forma um ângulo. Este ângulo relaciona-se com o tipo facial (mesofacial, dolicofacial e braquifacial).

Ângulo V - Ângulo formado pela intersecção das linhas que se estendem do ponto $\mathrm{V}$ ao gônio direito (God') e do ponto $V$ ao gônio esquerdo (Goe').

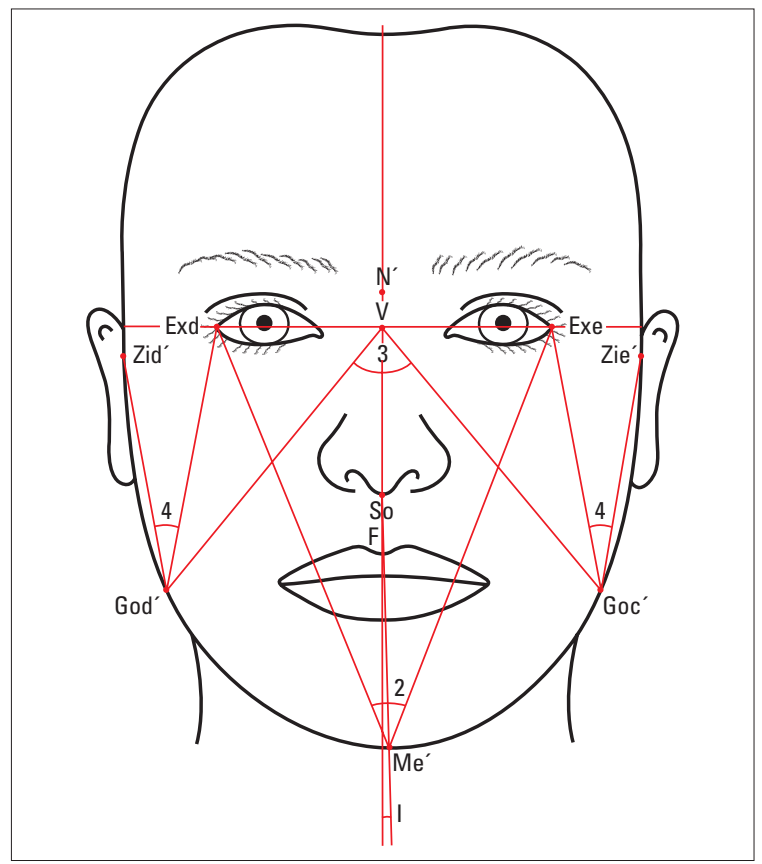

FIGURA 3 - Medidas fotométric as angulares.

01 - Ângulo da simetria facial

02 - Ângulo da abertura facial modificado

03 - Ângulo V

04 - Simetria entre o lado direito e esquerdo da face 


\begin{tabular}{|c|c|c|c|c|c|c|c|}
\hline VARIÁVEL & MÉDIA & MEDIANA & MÍNIMO & MÁXIMO & DESVIO PADRÃO & DP ERRO & COEF. DE VAR \\
\hline Comprimento do lábio sup. (Sn-Es) & 11,91 & 12,00 & 10,00 & 14,50 & 1,11 & 0,158 & 9,31 \\
\hline Comprimento do lábio inf. (Es-Me') & 23,73 & 23,50 & 21,00 & 28,00 & 1,79 & 0,224 & 7,55 \\
\hline Vermelhão do lábio sup. (Ls-Es) & 3,38 & 3,25 & 2,50 & 5,00 & 0,64 & 0,194 & 18,90 \\
\hline Vermelhão do lábio inf. (Es-Li) & 5,90 & 6,00 & 4,50 & 8,50 & 0,89 & 0,194 & 15,01 \\
\hline Altura da boca (Ls-Li) & 9,28 & 9,00 & 7,00 & 13,00 & 1,18 & 0,194 & 12,74 \\
\hline Largura da boca (Abd-Abe) & 27,01 & 27,00 & 24,00 & 31,50 & 1,74 & 0,250 & 6,43 \\
\hline Inclinação da linha da comissura & 0,55 & 0,50 & 0,00 & 2,50 & 0,57 & 0,224 & 104,57 \\
\hline Altura do terço médio (Gl’-Sn) & 34,23 & 34,25 & 30,50 & 38,00 & 1,77 & 0,250 & 5,17 \\
\hline Altura do terço inferior (Sn-Me') & 35,56 & 35,50 & 31,00 & 41,00 & 2,47 & 0,296 & 6,93 \\
\hline
\end{tabular}

\begin{tabular}{|c|c|c|c|c|c|c|c|}
\hline \multicolumn{10}{|c|}{ Tabela 2 - Medidas Angulares. } \\
\hline VARIÁVEL & MÉDIA & MEDIANA & MÍNIMO & MÁXIMO & DESVIO PADRÃO & DP ERRO & COEF. DE VAR \\
\hline Ângulo da simetria facial & 0,54 & 0,00 & 0,00 & 2,00 & 0,75 & 0,158 & 138,76 \\
\hline Âng. da abertura facial modificado & 43,75 & 44,00 & 39,50 & 46,00 & 1,51 & 0,224 & 3,45 \\
\hline Âng. V & 71,5 & 71,00 & 64,00 & 80,00 & 3,68 & 0,602 & 5,15 \\
\hline Simetria entre o lado direito e esquerdo da face & 1,30 & 1,25 & 0,00 & 4,00 & 0,98 & 0,475 & 75,83 \\
\hline
\end{tabular}

\begin{tabular}{|c|c|c|c|c|c|c|c|c|}
\hline \multicolumn{10}{|c|}{ Tabela 3 - Medidas Proporcionais. } \\
\hline VARIÁVEL & MÉDIA & MEDIANA & MÍNIMO & MÁXIMO & DESVIO PADRÃO & DP ERRO & COEF. DE VAR \\
\hline Índice facial & 85,38 & 85,65 & 78,60 & 95,00 & 3,19 & 0,413 & 3,73 \\
\hline Proporção Gl'-Sn e Sn-Me' & 1,04 & 1,01 & 0,94 & 1,19 & 0,63 & 0,041 & 6,11 \\
\hline
\end{tabular}

\begin{tabular}{|c|c|c|}
$\begin{array}{c}\text { Tabela } 4 \text { - Distribuição de Freqüência e Percentual do } \\
\text { Índice Facial. }\end{array}$ \\
\hline Í́NDICE FACIAL & FREO. & PERCENTUAL \\
\hline Braquifacial severo & 1 & $2,5 \%$ \\
\hline Braquifacial & 14 & $35 \%$ \\
\hline Mesofacial & 22 & $55 \%$ \\
\hline Dolicofacial & 2 & $5 \%$ \\
\hline Dolicofacial severo & 1 & $2,5 \%$ \\
\hline
\end{tabular}

Este ângulo relaciona-se com o tipo facial.

Simetria entre o lado direito e esquerdo da face - É a diferença entre as medidas dos ângulos direito e esquerdo formados pela intersecção da linha Zi'-Go' e a linha Ex-Go'.

\section{ANÁLISE ESTATÍSTICA}

Por meio da análise estatística, foram apresentadas medidas de posição e de dispersão para as variáveis contínuas das fotografias. Foram apresentadas as informações correspondentes à média amostral, mediana, valor mínimo observado, valor máximo observado, desvio padrão, erro padrão e coeficiente de variação.

A confiabilidade das medidas utilizadas neste trabalho foram avaliadas selecionando-se, aleatoriamente, as fotografias de 10 indivíduos entre os 40 , totalizando 20 fotografias: 10 fotografias em repouso e 10 durante o sorriso. Nessas fotografias, novos traçados e medições foram feitos. Para o cálculo do desvio padrão do erro foi utilizada a fórmula de 
Dahlberg ${ }^{31}$. Os erros acima de $1 \mathrm{~mm}$ para as medidas lineares e acima de $1,5^{\circ}$ para medidas angulares foram considerados como erros absolutos, altos e significantes.

\section{RESULTADOS}

Nas tabelas 1 a 4 são apresentadas, para as variáveis contínuas, as informações correspondentes à média amostral, mediana, valor mínimo observado, valor máximo observado, desvio padrão, desvio padrão do erro e coeficiente de variação.

\section{DISCUSSÃO}

Muito tem sido escrito sobre o conceito de beleza e estética facial, mas sua discussão dentro da Ortodontia é um tanto limitada, particularmente com relação à quantificação dos tecidos moles faciais de contorno.

Este trabalho analisa a face em uma visão frontal, por meio de traçados feitos sobre fotografias padronizadas utilizando-se de medidas lineares, angulares e proporcionais. A face é analisada em posição de repouso e durante o sorriso máximo.

A avaliação da estética facial é subjetiva e difícil, pois não existe uma estética ideal única.

Farkas e $\mathrm{Kolar}^{23}$ realizaram um trabalho com 34 mulheres de diferentes origens étnicas, consideradas de beleza acima da média. Dessas mulheres, oito foram consideradas como as mais belas em uma visão frontal. Para o grupo com faces atrativas obtiveram o valor de $20 \mathrm{~mm}$ para o comprimento do lábio superior e 19,1 mm nas mais atrativas. Segundo Psillakis e Lucardi ${ }^{41}$ o comprimento do lábio superior deve estar entre 17 e 23 mm. Peck, Peck e Kataja ${ }^{39}$, em estudo com adolescentes norte-americanas de origem européia, encontraram o valor médio de 21,2 mm. Para Arnett e Bergman ${ }^{1,2}$, o comprimento do lábio superior deve estar entre 19 e $22 \mathrm{~mm}$. Nanda e $\mathrm{Ghosh}^{37}$, estudando a face de mulheres jovens leucodermas, encontraram como valor médio para o lábio superior 20,96 mm. Em nosso estudo, encontramos a média de $21,64 \mathrm{~mm}$, concordando com as médias obtidas pelos outros autores.
Segundo Psillakis e Lucardi ${ }^{41}$, o comprimento do lábio inferior deve estar entre 38 e $52 \mathrm{~mm}$ e para Arnett e Bergman ${ }^{1,2}$, entre 38 e $44 \mathrm{~mm}$. Farkas e $\operatorname{Kolar}^{23}$, obtiveram o valor médio de $43,6 \mathrm{~mm}$ para as faces atrativas e $42,1 \mathrm{~mm}$ para as faces mais atrativas. $\mathrm{O}$ valor médio encontrado em nossa amostra foi de $43,15 \mathrm{~mm}$, muito próximo do encontrado na literatura.

O valor médio obtido para a altura da boca, em nosso trabalho, foi de $16,87 \mathrm{~mm}$, próximo do valor médio de 17,66 mm encontrado por Ferrario et al. ${ }^{24}$.

Quanto à largura da boca, Farkas e outros ${ }^{22}$ encontraram $50 \mathrm{~mm}$. Farkas e Kolar ${ }^{23}$ encontraram, nas faces atrativas, $50,8 \mathrm{~mm}$ e, nas mais atrativas, 51 $\mathrm{mm}$. Rigsbee III, Sperry e Begole ${ }^{43}$ numa amostra mista formada por mulheres tratadas ortodonticamente e não tratadas, obtiveram uma média de 49,89 mm. Epker ${ }^{19}$ obteve o valor médio de 55 $\mathrm{mm}$. Ferrario et al. ${ }^{24}$ obtiveram $47,38 \mathrm{~mm}$. Ferrario et al. ${ }^{26}$, no primeiro grupo, obtiveram $52,4 \mathrm{~mm}$ e no segundo, 50,96 mm. Nanda e Ghosh ${ }^{37}$ obtiveram 47,04 mm. Moss, Liney e Lowey ${ }^{35}$ obtiveram $48,5 \mathrm{~mm}$. Nossa amostra apresentou uma média de $49,11 \mathrm{~mm}$, próximo aos valores médios encontrados na literatura.

A inclinação da linha da comissura nos dá uma noção de simetria da boca em relação à face através do paralelismo existente entre a linha interexocanto e a linha da comissura. Em nossa amostra a média foi de $1,0 \mathrm{~mm}$, o que demonstra um equilíbrio facial compativel com a normalidade.

Farkas $^{22}$, estudando adultos jovens, encontrou a média de $8,4 \mathrm{~mm}$ para a altura do vermelhão do lábio superior e $9,7 \mathrm{~mm}$ para a altura do vermelhão do lábio inferior. Moss, Liney e Lowey ${ }^{35}$ encontraram a média de $8,8 \mathrm{~mm}$ e 9,5 $\mathrm{mm}$. Em nossa amostra, o valor médio é de $6,15 \mathrm{~mm}$ e $10,73 \mathrm{~mm}$, respectivamente. Isso nos leva a concluir que o lábio superior é mais fino na amostra em questão.

Segundo Arnett e Bergman ${ }^{1,2}$, o terço médio e inferior da face deverão ser proporcionais e variando entre 55 e $65 \mathrm{~mm}$. Para o terço inferior da 
face, Farkas et al..$^{22}$ obtiveram $66 \mathrm{~mm}$. Farkas e Ko$\operatorname{lar}^{23}$ obtiveram, nas faces atrativas, 64,9 mm e 62,6 $\mathrm{mm}$ nas mais atrativas. Em nosso trabalho, a altura do terço médio foi de $62,24 \mathrm{~mm}$ e a altura do terço inferior foi de $64,65 \mathrm{~mm}$, muito próxima dos valores médios encontrados por outros autores. A razão entre o terço médio e inferior da face foi de 1,04. Alguns autores consideram o terço médio da face a partir do násio até o subnasal ${ }^{18,41}$. Em nosso trabalho, consideramos o terço médio da face, da glabela ao subnasal.

No alinhamento da ponta do nariz e do mento (ponto mentoniano) com a linha média facial tivemos uma coincidência em 21 fotografias. A ponta do nariz coincidiu com a linha média facial em 31 fotografias, em 9 fotografias o desvio variou de $0,5 \mathrm{~mm}$ a $1,0 \mathrm{~mm}$. O ponto mentoniano coincidiu com a linha média facial em 24 fotografias e em 16 fotografias o desvio variou de $0,5 \mathrm{~mm}$ a 1,5 $\mathrm{mm}$. A ponta do nariz teve uma média de desvio de $0,27 \mathrm{~mm}$ em relação à linha média facial e o ponto mentoniano, $0,84 \mathrm{~mm}$.

O ângulo da simetria facial, que avalia o desvio do mento no sentido transversal, teve uma média de $0,54^{\circ}$, indicando que, na amostra estudada, o desvio do mento é mínimo e a assimetria é pouco significante. Este ângulo pode ser comparado ao ângulo formado entre a linha mediana maxilo-mandibular (Ena-Me') e o plano sagital mediano (plano perpendicular à linha zigomática superior (ZSD-ZSE) passando pela espinha nasal anterior) na análise facial frontal em telerradiografias póstero-anterior.

Segundo Viazis ${ }^{49}$ o ângulo da abertura facial deve ser de $45^{\circ}$ com uma variação de mais ou menos $5^{\circ}$. Valores maiores deste ângulo indicariam uma face mais larga, mais quadrada e, valores menores uma face mais longa, estreita. $\mathrm{O}$ autor utiliza duas linhas que se estendem desde o canto externo da órbita do lado direito e esquerdo, passando pela união dos lábios superior e inferior nos cantos da boca ${ }^{49}$. A intersecção dessas linhas forma o ângulo da abertura facial. Em nosso trabalho modificamos os pontos anatômicos preconizados por Viazis ${ }^{49}$. O canto externo da órbita é substituído pelo exocanto, devido a dificuldade de uma visualização precisa. O ponto na união do lábio inferior com o lábio superior nos cantos da boca é substituído pelo ponto mentoniano. A média encontrada para a amostra foi de $43,75^{\circ}$.

$\mathrm{O}$ ângulo $\mathrm{V}$, ângulo formado pela intersecção das linhas da direita e da esquerda que se estendem desde o ponto $\mathrm{V}$ até o gônio direito (God') e esquerdo (Goe'), teve um valor médio de $71,5^{\circ}$. Esse ângulo relaciona-se com o tipo facial. Um indivíduo com uma face longa terá uma diminuição nesse ângulo e uma face curta terá um aumento. Novos estudos deverão ser feitos, com os diferentes tipos faciais, para determinar o valor médio para cada um deles (meso, dólico e braquifacial).

A simetria entre o lado direito e esquerdo da face, medida por meio da diferença entre os ângulos formados pela linha Ex-Go' e a linha Zy'-Go' do lado direito e esquerdo da face, teve uma média de $1,3^{\circ}$, revelando faces clinicamente simétricas. Esta medida pode ser comparada com a simetria postural utilizada na análise facial frontal em telerradiografias póstero-anterior.

Para o índice facial, Farkas ${ }^{22}$ obteve em sua amostra a média de 86,2. Farkas e Kolar ${ }^{23}$ encontraram nas faces atrativas a média de 86,6 e nas mais atrativas, 85,7. Em nosso trabalho, obtivemos uma média de 85,38 , o que indica que a média da amostra foi mesofacial e, aproximadamente, a mesma média encontrada pelos outros autores. Do total da amostra, 2,5\% eram braquifacial severo, $35 \%$ braquifacial, 55\% mesofacial, 5\% dolicofacial e 2,5\% dolicofacial severo.

A média da proporção entre a altura da face média e a altura da face inferior foi de 1:1,04. Segundo os padrões clássicos de proporcionalidade facial, a proporção ideal é de $1: 1$, estando pois a amostra muito próxima do ideal.

Cummins, Bishara, Jakobsen ${ }^{15}$ testaram a confiabilidade das medidas fotogramétricas. Os resultados mostraram que em $17 \%$ das medidas feitas pelo mesmo examinador o erro foi maior que $1 \mathrm{~mm}$ 


\begin{tabular}{|c|c|c|}
\hline Variável & $\begin{array}{c}\text { Média Obtida } \\
\text { na Fotografia }\end{array}$ & $\begin{array}{c}\text { Medida } \\
\text { "Real" }\end{array}$ \\
\hline Comprimento do lábio sup. (Sn-Es) & 11,91 & 21,64 \\
\hline Comprimento lábio inf.-mento (Es-Me') & 23,73 & 43,15 \\
\hline Ext. do vermelhão do lábio sup. (Ls-Es) & 3,38 & 6,15 \\
\hline Ext. do vermelhão do lábio inf. (Es-Li) & 5,90 & 10,73 \\
\hline Altura da boca (Ls-Li) & 9,28 & 16,87 \\
\hline Inclinação da linha da comissura & 0,55 & 1,00 \\
\hline Altura do terço médio (Gl'-Sn) & 34,23 & 62,24 \\
\hline Altura do terço inferior (Sn-Me') & 35,56 & 64,65 \\
\hline Largura da boca (Abd-Abe) & 27,01 & 49,11 \\
\hline Alinham. da Lmf-ponta do nariz & 0,15 & 0,27 \\
\hline Alinham. da Lmf-ponta do mento & 0,46 & 0,84 \\
\hline Ângulo da simetria facial & 0,54 & 0,54 \\
\hline Ângulo da abertura facial modificado & 43,75 & 43,75 \\
\hline Ângulo V & 71,50 & 71,50 \\
\hline Simetria entre o lado direito & 1,30 & 1,30 \\
\hline e esquerdo da face & & 85,38 \\
\hline Índice facial & 85,38 & 1,04 \\
\hline Proporção Gl'-Sn e Sn-Me' & 1,04 & \\
\hline
\end{tabular}

QUADRO 1 - Fotografia frontal em repouso.

ou $1^{\circ}$. Para Bishara, Jorgensen, Jakobsen ${ }^{8,9}$ a magnitude do erro intra-examinador foi de $0,5 \mathrm{~mm}$ ou menos em $79 \%$ das medidas, e $96 \%$ das medidas apresentaram menos de $1 \mathrm{~mm}$ de diferença. Bishara et al. ${ }^{7}$, relataram, em seu estudo, que nas fotografias frontais, o comprimento do lábio inferior (Es-Me') foi menos confiável que as outras medidas, devido à dificuldade de localização do ponto mentoniano. A largura da boca também mostrou relativamente mais variabilidade devido às sombras do ângulo da boca ou devido ao movimento do lábio. Para Strauss e outros ${ }^{46}$ a margem clínica aceitável para a reprodutibilidade foi de $1 \mathrm{~mm}$ em medidas lineares e $2^{\circ}$ em medidas angulares.

Em nosso estudo, o teste de confiabilidade foi realizado pelo mesmo examinador utilizando o desvio padrão do erro ${ }^{31}$, e não ultrapassou $1 \mathrm{~mm}$ em $100 \%$ das medidas lineares. Com relação às medidas angulares, tivemos um valor maior do desvio padrão do erro $^{31}$ para algumas medidas que envolviam os pontos gônio tegumentar (direito e esquerdo)

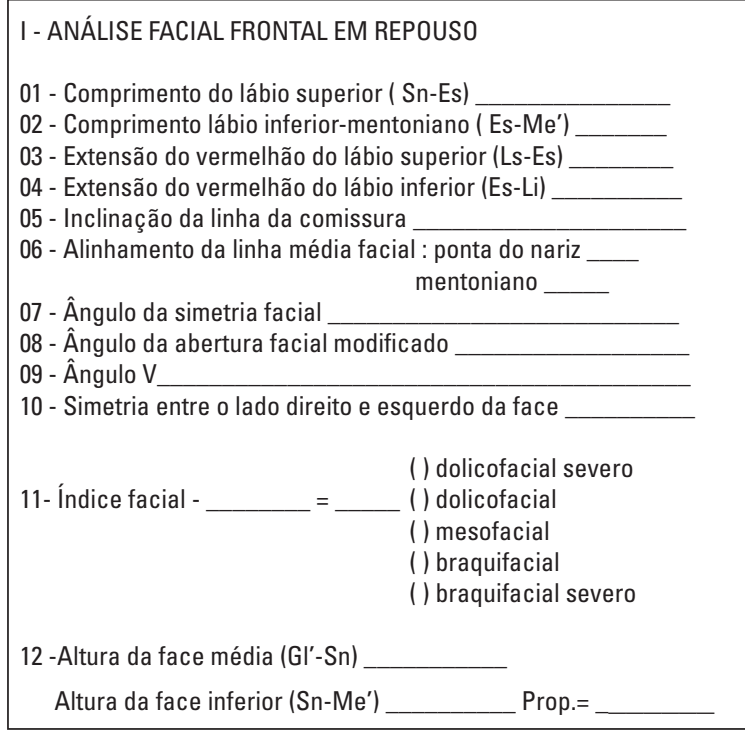

QUADRO 2 - Análise facial em repouso.

devido à dificuldade na determinação deste ponto, mas nenhuma delas ultrapassou $1,5^{\circ}$. Portanto, as medidas utilizadas em nossa análise são confiáveis.

A análise facial em fotografias frontais padronizadas pode ser utilizada como auxiliar importante no diagnóstico e planejamento do tratamento ortodôntico e cirúrgico, permitindo uma avaliação quantitativa da face antes e durante o tratamento, bem como apresentando-se como um método confiável para comparações entre o pré e o pós-tratamento. Entretanto, é importante ressaltar que ela não dispensa a avaliação clínica e radiográfica da face do paciente.

\section{CONCLUSÕES}

Após a exposição e discussão dos resultados, parece lícito concluir que:

1) Os padrões médios de normalidade para as variáveis da análise da fotografia frontal em repouso da amostra estudada foram mensurados (Quad. 1).

2) Algumas das medidas utilizadas em nosso 
trabalho se assemelham às encontradas na literatura e outras diferem muito. A origem da amostra utilizada e a metodologia colaboraram para esta divergência de resultados. Com uma padronização da metodologia, como sugerida em nosso trabalho, podemos fazer comparações de trabalhos com amos- tras da mesma origem étnica e de origens étnicas diferentes.

3) Todas a variáveis mostraram-se confiáveis após a repetição das medições.

4) Proposição de uma análise facial frontal em fotografias. (Quad. 2).

\title{
Facial frontal analysis in rest position and maximun smile in standardized photoghaphs
}

\begin{abstract}
Considering the importance of the frontal facial analysis in the diagnosis, the purpose of this research is to present a frontal facial analysis using standardized photographs, in rest position and during the smile, as an aid in the diagnosis and planning of the orthodontic and surgical treatment as well as in the evaluation of treatment results. The sample consisted of 40 white female subjects with a mean age of 22 years old, pleasant face, normal occlusion, and had not been previously submitted to orthodontic treatment nor plastic surgery. Colorful standardized 10x15 cm photographs were obtained with the patients in their natural head position. Two pictures were taken for each subject, one in maximum smile and another in rest position. Linear, angular and proportional measurements were used. Tests of normality, descriptive statistics, and error standard deviation were executed. The results showed that some of the measurements used in this study have values similar to that found in the literature and some are very different. All of the measurements were reliable after their repetition. And after this study, it is suggested a frontal facial analysis to be used regularly in the diagnosis and orthodontic treatment planning.
\end{abstract}

Key words: Facial frontal analysis. Orthodontics. Facial aesthetics.

\section{REFERÊNCIAS}

1. ARNETT, W. G.; BERGMAN, R. T. Facial keys to orthodontic diagnosis and treatment planning: part l. Am J Orthod Dentofacial Orthop, St. Louis, v.103, no. 4, p. 299-312, Apr. 1993.

2. ARNETT, W. G.; BERGMAN, R. T. Facial keys to orthodontic diagnosis and treatment planning - part II. Am J Orthod Dentofacial Orthop, St. Louis, v.103, no. 5, p. 395-441, May 1993.

3 AYOUB, F. A.; WRAYM, D.; MOOS, K. F. Three-dimensional modeling for modern diagnosis and planning in maxillofacial surgery. Int J Adult Orthodon Orthognath Surg, Lombard, v. 11, no. 3, p. 225-233, 1996.

4. BARNETT, A. N.; WHITAKER, L. A. Facial form analysis of the lower and middle face. Plast Reconst Surg, Baltimore, v. 78, no. 2, p. 158-165, Aug. 1986.

5 BELINFANTE, L. S. Total treatment planning for esthetic problems of the face: a team approach. J Oral Surg, Chicago, v. 37, Mar. 1979.

6. BENGEL, W. Standardization in dental photography. Int Dent J, New York, v. 35, no. 3, p. 2100-2117, Sept. 1985.
7. BISHARA, S. E. et al. A computer assisted photogrammetric analysis of soft tissue changes after orthodontic treatment. Part I: methodology and reliability. Am J Orthod Dentofacial Orthop, St. Louis, v.107, no. 6, p. 633-639, June 1995.

8. BISHARA, S. E.; JORGENSEN, G. J.; JAKOBSEN, J. R. Changes in facial dimensions assessed from lateral and frontal photographs. Part I - Metodology. Am J Orthod Dentofacial Orthop, St. Louis, v.108, no. 4, p. 389-393, Oct. 1995.

9. BISHARA, S. E.; JORGENSEN, G. J.; JAKOBSEN, J. R. Changes in facial dimensions assessed from lateral and frontal photographs. Part II - Results and conclusions. Am J Orthod Dentofacial Orthop, St. Louis, v. 108, no. 5, p. 489-499, Nov. 1995.

10. CALTABIANO, M.; VERZI, P. Valutazione delle asimmetrie facciali com una nuova metodica mediant tracciato su fotografia frontale. Minerva Stomatol, Torino, v. 34, p. 853-857, 1985.

11. CHIU, C. S. W.; CLARK, R. K. F. Reproducibility of natural head position. J Dent, Chengtu, v. 19, p.130-131, Jan. 1991.

12. CLAMAN, L.; PATTON, D.; RASHID, R. Standardized portrait 
photography for dental patients. Am J Orthod Dentofacial Orthop, St. Louis, v. 98, no. 3, p. 197-205, Sept. 1990.

13. COOKE, M. S. Five-year reproducibility of natural head posture: a longitudinal study. Am J Orthod Dentofacial Orthop St. Louis, v. 97, no. 6, June 1990

14. COOKE, M. S.; WEI, S. H. Y. The reproducibility on natural head posture: A methodological study. Am J Orthod Dentofacial Orthop, St. Louis, v. 93, no. 4, p. 280-288, Apr. 1988

15. CUMMINS, D. M.; BISHARA, S. E.; JAKOBSEN, J. R. A computer assisted photogrammetric analysis of soft tissue changes after orthodontic treatment. Part II: Results. Am J Orthod Dentofacial Orthop, St. Louis, v. 108, no. 1, p. 38-47, July 1995

16. DIERKES, J. M. The Beauty of the face: an orthodontic perspective. J Am Dent Assoc, Chicago, v. 7, p. 35-43, Dec. 1987.

17. DUBRUL, L. E. Sicher's oral anatomy. 7th ed. St. Louis: Mosby, 1980 .

18. EL-MANGOURY, N. H.; MOSTAFA, Y. A. Faciometrics: A new syntax for facial feature analysis. Int J Adult Orthod Orthognath Surg, Lombard, v. 11, no.1, 1996.

19. EPKER, B. N. Adjuntctive esthetic surgery in the orthognathic surgery patient. In: McNAMARA Jr., J. A. Esthetic and the treatment of facial form. Ann Arbor: University of Michigan; Center for Human Growth and Development 1992. p.18-35. (Craniofacial Growth Series, v. 28).

20. FARKAS, L. G. Antropometry of the head and face in medicine. New York: Elsevier Science, 1981

21. FARKAS, L. G.; BRYSON, W.; KLOTZ, J. Is photogrammetry of the face reliable? Plast Reconstr Surg, Baltimore, v. 66, no. 3, p. 36-45, Sept. 1980

22. FARKAS, L. G. et al. Vertical and horizontal proportions of the face in young adult North American Caucasians: revision of neoclassics canons. Plast Reconstr Surg, Baltimore, v. 75, no. 3, p. 328-338, Mar. 1985

23. FARKAS, L. G.; KOLAR, J. C. Anthropometrics and art in the aesthetics of Women's faces. Clin Plastic Surg, Philadelphia, v.14, no. 4, p. 599-516, Oct. 1987

24. FERRARIO, V. F. et al. Craniofacial morphometry by photographic evaluations. Am J Orthod Dentofacial Orthop, St. Louis, v.103, no. 4, Apr. 1993

25. FERRARIO, V. F. A three-dimensional study of sexual dimorphism in the human face. Int J Adult Orthod Orthognath Surg, Lombard, v. 9, no. 4, p. 303-310, 1994.

26. FERRARIO, V. F. et al. A three-dimensional evaluation of human facial asymmetry. J Anat, London, v. 186, p. 103-110, 1995.

27. FERRARIO, V. F. et al. Facial morphometry of television actresses compared with normal women. J Oral Maxilofac Surg Philadelphia, v. 53, p.1008-1014, 1995

28. FRICKER, J. P. Standardized facial photography. Austr Orthod J, Brisbane, v.7, p.168-173, Oct. 1982

29. GONZÁLEZ-ULLOA, M. A quantum method for the appreciation of the morphology of the face. Plast Reconstr Surg, Baltimore, v. 34, no. 3, p. 241-246,1964.

30. HERZBERG, B. L. Facial esthetic in relation to orthodontic treatment. Angle Orthod, Appleton, v. 22, no.1, p. 3-22, Jan. 1952

31. HOUSTON, W. J. B. The analysis of errors in orthodontic measurements. Am J Orthod, St. Louis, v. 83, no. 5, p. 382-390, May 1983.

32. MACKLEY, R. J. Development of balance and harmony of the smile. Func Orthod, Winchester, v.10, no.3, p. 5-21, May/June 1993.
33. McNAMARA Jr., J. A. BRUST, E. W. RIOLO, M. L Soft tissue evaluation of individuals with an ideal occlusion and a wellbalanced face. In: McNAMARA Jr., J. A. Esthetic and the treatment of facial form. Ann Arbor: University of Michigan. Center for Human Growth and Development. 1992. p. 185-212. (Craniofacial Growth Series, v.28).

34. MOORREES, C. F. A. Natural head position: a reviwel. Am J Orthod Dentofacial Orthop, St. Louis, v. 105, no. 5, p. 512-513, May 1994.

35. MOSS, J. P.: LINNEY, A D.: LOWEY, M. N. Uso das técnicas tridimensionais na estética facial. In: SADOWSKY, P. L. et al. Atualidades em Ortodontia. São Paulo: Premier, 1997. p. 89-97

36. MOTOYOSHI, M.; NAMURA, S.; ARAI, A. Y. A three-dimensional measuring system for the human face using three-dimensional photography. Am J Orthod Dentofacial Orthop, St. Louis, v.101, no. 5, p. 431-440, May 1992.

37. NANDA, R. S. GHOSH, J. Harmonia entre os tecidos moles da face e o crescimento no tratamento ortodôntico. In: SADO WSKY, P. L. et al. Atualidades em Ortodontia. São Paulo: Premier, 1997. p. 65-78.

38. PECK, S.; PECK, L. Aspectos selecionados da arte e da ciência da estética facial. In: SADOWSKY, P. L. et al. Atualidades em Ortodontia. São Paulo: Premier, 1997. p. 99-116.

39. PECK, S.; PECK, L.; KATAJA, M. Some vertical lineaments of lip position. Am J Orthod Dentofacial Orthop, St. Louis, v.101, no. 6, p. 519-524, June 1992

40. PHILLIPS, C. et al. Photocephalometry: Errors of projection and landmark location. Am J Orthod, St. Louis, v. 84, p. 233-243 Sept. 1984.

41. PSILLAKIS, J. M.; LUCARDI, V. Proporções estéticas da face. In: PSILLAKIS, J. M. et al. Cirurgia craniomaxilofacial: osteotomias estéticas da face. Rio de Janeiro: Medsi, 1987. p. 297-309.

42. RICKETTS, R. M. The biologic significance of the divine proportion and Fibonacci series. Am J Orthod, St. Louis, v. 81, no. 5 , p. 351-370, May 1982

43. RIGSBEE III, O. H.; SPERRY, T. P.; BEGOLE, E. A. The influence of facial animation on smile characteristics. Int J Adult Orthod Orthognath Surg, Lombard, v. 3, p. 233-239, 1988.

44. ROBERTSON, N. R. E.; VOLP, C. R. Telecentric photogrammetry: its development, testing, and application. Am J Orthod Dentofacial Orthop, St. Louis, v. 8, p. 623-637, Dec. 1981.

45. SOUZA, A. M. Estudo esquemático de fotografias em rinoplastias. R Latino Americana Cirurgia Plástica, São Paulo, v. 8, n. 3, p. 31-43, set. 1964.

46. STRAUSS, R. A. et al. Variability of facial photographs for use in treatment planning for orthodontics and orthognathic surgery. Int J Adult Orthod Orthognath Surg, Lombard, v.12, no. 3 p. 197-203, 1997.

47. SUGUINO, R. et al. Análise facial. R Dental Press de Ortodon Ortop Facial, Maringá, v. 1, n. 1, p. 86-107, set./out. 1996.

48. VIAZIS, A. D. A cephalometric analysis based on natural head position. J Clin Orthod, Boulder, v. 25, no. 3, p.172-181, Mar. 1991.

49. VIAZIS, A. D. Avaliação do tecido mole. In: Atlas de Ortodontia: princípios e aplicações clínicas. São Paulo: Ed Santos, 1996. p. 49-57

50. ZAREM, H. A. Standards of photography. Plast Reconstr Surg Baltimore, v. 74, no. 1, p.137-144, July 1984

Endereço para correspondência

Alexandre Moro

Av. Silva Jardim, 2675 Água Verde

Curitiba-PR. Brasil.

CEP: 80240-020

e-mail: amoro@bio.ufpr.br 